\title{
Two Scrolls or One Click: A Cost Model for Browsing Search Results
}

\author{
Leif Azzopardi ${ }^{1}$ and Guido Zuccon ${ }^{2}$ \\ 1 School of Computing Science, University of Glasgow \\ leif .azzopardi@glasgow.ac.uk \\ 2 Information Systems School, Queensland University of Technology (QUT) \\ g.zuccon@qut.edu.au
}

\begin{abstract}
Modeling how people interact with search interfaces has been of particular interest and importance to the field of Interactive Information Retrieval. Recently, there has been a move to developing formal models of the interaction between the user and the system, whether it be to run a simulation, conduct an economic analysis, measure system performance, or simply to better understand the interactions. In this paper, we present a cost model that characterizes a user examining search results. The model shows under what conditions the interface should be more scroll based or more click based and provides ways to estimate the number of results per page based on the size of the screen and the various interaction costs. Further extensions to the model could be easily included to model different types of browsing and other costs.
\end{abstract}

\section{Introduction}

An emerging area of research within Interactive Information Retrieval (IIR) is the development of cost models that characterize the cost of interaction with a particular interface. Such cost models have been used for various purposes, such as: (i) controlling the number of interactions a simulated user can perform in a given period of time $[4,5]$, (ii) approximating the time spent examining the results in a ranked list, e.g., within time biased measures [11,12], (iii) analyzing and empirically evaluating the costs and benefits of different interfaces $[2,8,10]$ (iv) estimating the cost of different courses of interaction to determine the most efficient course of action $[1,7,9]$. Essentially, these lines of research have aimed at measuring the cost that the user incurs, as opposed to traditional performance measures, which look at measuring the gain or benefit that the user receives from such actions. Thus such models/measures of cost complement and extend the existing research on measuring the value of searching from a user perspective [3].

In this paper, we will focus on developing a cost model that captures the costs of a user interacting with a ranked list by browsing through the list until they find the document they would like to inspect. We use this model to estimate how many results per page should be shown under particular conditions. 


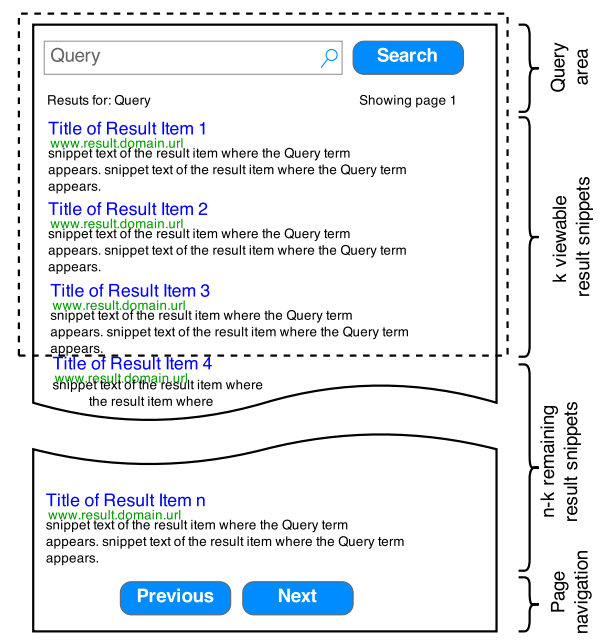

Fig. 1. The area marked by the dotted line shows how much of the page is initially visible, where $\mathbf{k}$ snippets can be seen. $\mathbf{k}$ will vary according to screen size. If the number of results per page $\mathbf{n}$ is larger than $\mathbf{k}$, then $\mathbf{n}-\mathbf{k}$ results are below the fold.

\section{Cost Model}

To develop a cost model for results browsing we assume that the user will be interacting with a standard search engine result page (SERP) with the following layout: a query box, a list of search results (snippets), and pagination buttons (see Figure 1). Put more formally, the SERP displays $\mathbf{n}$ snippets, of which only $\mathbf{k}$ are visible above-the-fold. To view the remaining $\mathbf{n}-\mathbf{k}$ snippets, i.e., those that are below-the-fold, the user needs to scroll down the page, while to see the next $\mathbf{n}$ snippets, the user needs to paginate (i.e., click next). And so we wonder whether is it better to scroll, click, or some combination of?

Here, we consider the case where the user wants the document at the mth result. However, $\mathbf{m}$ is not known a priori. To calculate the total browsing costs we assume that the user has just entered their query and has been presented with the result list. We further assume that there are three main actions the user can perform: inspecting a snippet, scrolling down the list, or clicking to go to the next page. Therefore, we are also assuming a linear traversal of the ranked list. Each action incurs a cost: $\mathbf{C}_{\mathbf{s}}$ to inspect a snippet, $\mathbf{C}_{\mathbf{s c r}}$ to scroll to the next snippet ${ }^{3}$, and when the user presses the 'next' button to see the subsequent $\mathbf{n}$ results, they incur a click cost $\mathbf{C}_{\mathbf{c}}$. The click cost includes the time it takes the user to click and the time it takes the system to respond. Given these costs, we can now express a cost model for browsing to the mth result as follows:

$$
C_{b}(n, k, m)=\underbrace{\left\lfloor\frac{m}{n}\right\rfloor \cdot C_{c}}_{\text {clicking }}+\underbrace{\left(\left\lfloor\frac{m}{n}\right\rfloor \cdot(n-k)+\left(m_{r}-k\right)\right) \cdot C_{s c r}}_{\text {scrolling }}+\underbrace{m \cdot C_{s}}_{\text {inspecting }}
$$

\footnotetext{
$\overline{3}$ i.e., the scroll cost is the average cost to scroll the distance of one snippet, which includes the time to scroll and then focus on the next result.
} 
where $\mathbf{m}_{\mathbf{r}}$ represents the remaining snippets to inspect on the last page. Equation 1 is composed by three distinct components: the number of clicks the user must perform, the amount of scrolling required, and finally the number of snippets they need to inspect. The number of clicks is the number of pages that need to be viewed rounded up, because the whole page needs to be viewed. The number of scrolls is based on how many full pages of results need to be examined, and how many results remain on the last page that need to be inspected. The remaining snippets to inspect on the last page is: $\mathbf{m}_{\mathbf{r}}=\left(\mathbf{m}-\left\lfloor\frac{\mathbf{m}}{\mathbf{n}}\right\rfloor \mathbf{n}\right)$. However, if $\mathbf{m}_{\mathbf{r}}<\mathbf{k}$, then $\mathbf{m}_{\mathbf{r}}=\mathbf{k}$ as there is no scrolling on the last page. Note that $\mathbf{k}$ is bounded by $\mathbf{n}$, i.e., if only two results are shown per page, the maximum number of viewable results per page is 2 . It is possible that only part of the result snippet is viewable, so $\mathbf{k}$ is bound as follows: $\mathbf{0}<\mathbf{k} \leq \mathbf{n}$. The estimated cost is based on the number of "clicks," "scrolls" and result snippets viewed (i.e., m).

\subsection{Application and Example}

With this model it is possible to analyze the costs of various designs by setting the parameters accordingly. For example, a mobile search interface with a small screen size can be represented with a low $\mathbf{k}$, while a desktop search interface with a large screen can be characterized with a larger $\mathbf{k}$. The interaction costs for different devices can also be encoded accordingly.

Figure 2 shows an example of the cost of browsing $\mathbf{m}$ results when the number of results presented per page $(\mathbf{n})$ is $1,3,6$ and 10 with up to $k=6$ viewable results in the display window. Here we have approximated the costs of interaction as follows: 0.25 seconds to scroll, 2 seconds to click and 2.5 seconds to inspect a snippet $^{4}$.

Intuitively, displaying one result per page requires the most time as $(\mathbf{m}-\mathbf{1})$ clicks are needed to find the $\mathbf{m}$ th document. Displaying three results per page is also costly as $\mathbf{m}$ increases requiring approximately $\mathbf{m} / \mathbf{3}$ clicks. However, the difference in costs for 6 and 10 results per page vary depending on the specific number of results the user wants to inspect. For example, if $\mathbf{m}=\mathbf{1 2}$ then 6 results per page is lower in cost; whereas if $\mathbf{m}=\mathbf{1 3}$ then 10 results per page is lower in cost. In this example, since scrolling is relatively cheap, one might be tempted to conclude that the size of the result page should be as large as possible. However, using the model, we can determine the optimal size of the result page depending on the different parameters.

\subsection{Estimating SERP Size}

To determine the optimal number of results to show per page, $\mathbf{n}^{*}$, we want to minimize $\mathbf{C}_{\mathbf{b}}$. We can do this by differentiating the cost function with respect to

\footnotetext{
${ }^{4}$ These values were based on the estimated time spent examining each snippet being between 1.7 and 3 seconds from [1], the GOMS timings for a mouse move (1.1s), click (0.2s) and system response $(0.8 \mathrm{~s})$ being approximately 2 seconds taken from [2] and based on [6]. For the scroll action on a wheeled mouse, we assume that it is similar to a key press $(0.28 \mathrm{~s})$ and was approximated by 0.25 seconds.
} 


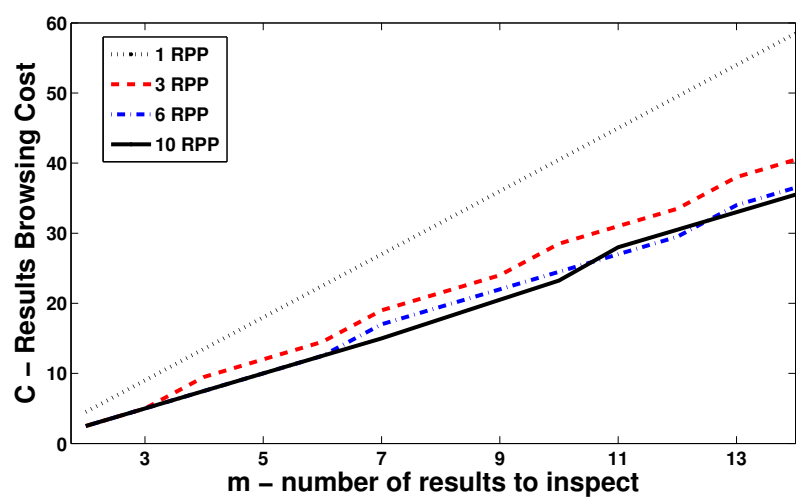

Fig. 2. The cost (total time in seconds) to examine $m$ snippets for SERPs of different sizes $\left(c_{s c r}=0.25, c_{c}=2, c_{s}=2.5\right.$ seconds $)$.

n. However, since $\mathbf{C}_{\mathbf{b}}$ contains floor operators we need to use an approximation of Eq. 1:

$$
\hat{C}_{b}(n, k, m) \approx \frac{m}{n} \cdot C_{c}+\frac{m}{n} \cdot(n-k) \cdot C_{s c r}+C_{s} \cdot m
$$

which essentially provides a smoothed estimate of the clicking and scrolling costs (here we drop/ignore the inspect costs as they are constant). We can now differentiate this function, to obtain:

$$
\frac{\partial \hat{C}_{b}}{\partial n}=-\frac{m}{n^{2}} \cdot C_{c}+\frac{m}{n^{2}} \cdot k \cdot C_{s c r}
$$

and then solve the equation by setting $\frac{\partial \hat{C}_{b}}{\partial n}=0$, in order to find what values minimize Eq. 2. The following is obtained:

$$
\begin{aligned}
-\frac{m}{n^{2}} \cdot C_{c}+\frac{m}{n^{2}} \cdot k \cdot C_{s c r} & =0 \\
\frac{m}{n^{2}} \cdot k \cdot C_{s c r} & =\frac{m}{n^{2}} \cdot C_{c} \\
k \cdot C_{s c r} & =C_{c}
\end{aligned}
$$

Interestingly, $\mathbf{n}$ disappears from the equation. This at first seems counter intuitive, as it suggests that to minimize the cost of interaction $\mathbf{n}$ is not a factor. However, on closer inspection we see that the number of results to show per page depends on the balance between $\mathbf{k}$ and $\mathbf{C}_{\mathbf{s c r}}$, on one hand, and $\mathbf{C}_{\mathbf{c}}$, on the other. If $\mathbf{k} . \mathbf{C}_{\mathbf{s c r}}$ is greater than, equal to, or less than $\mathbf{C}_{\mathbf{c}}$, then the influence on total cost in Equation 1 results in three different cases (see below). To help illustrate these cases, we have plotted three examples in Figure 3, where the user would like to inspect $\mathbf{m}=\mathbf{2 5}$ result snippets, and a maximum of $\mathbf{k}=\mathbf{6}$ result snippets are viewable per page.

1. if $\mathbf{k} \cdot \mathbf{C}_{\mathbf{s c r}}>\mathbf{C}_{\mathbf{c}}$, then $\mathbf{n}$ should be set to $\mathbf{k}$. In fact, if $\mathbf{n}=\mathbf{k}$, then $\mathbf{C}_{\mathbf{s c r}}$ has no effect on Equation 1, leaving only the inspecting and clicking costs. 
Intuitively, this means that if scrolling is expensive, then it is better to paginate rather than scroll. In Figure 3, the black dotted line shows this case, where the cost of larger size pages leads to greater total cost.

2. if $\mathbf{k} . \mathbf{C}_{\mathbf{s c r}}=\mathbf{C}_{\mathbf{c}}$, then any value of $\mathbf{n}$ will result in a similar total cost, as long as $\mathbf{n} \geq \mathbf{k}$. The intuition here is that since these costs are balanced, then the number of results per page is invariant of the costs, and so scroll based or click based solutions are equivalent. In Figure 3, the blue dot dash line shows when k. $\mathbf{C}_{\text {scr }} \approx \mathbf{C}_{\mathbf{c}}$.

3. finally, if $\mathbf{k} . \mathbf{C}_{\mathbf{s c r}}<\mathbf{C}_{\mathbf{c}}$, then $\mathbf{n}$ could be arbitrarily large. This is because the total cost of interaction (Equation 1) will then be dominated by the clicking cost. Intuitively, this means that if clicking is expensive, then it is better to scroll, rather than click; thus this advocates a solution such as infinite scrolling. In Figure 3, the red solid line shows an example of this case, where increasing the size of pages leads to a lower total cost overall according to our model, where the total cost decreases at a diminishing rate. However, it is likely that other costs, which have not been modelled (i.e., cognitive costs, download costs, etc.) would lead to an increase in total cost at some point.

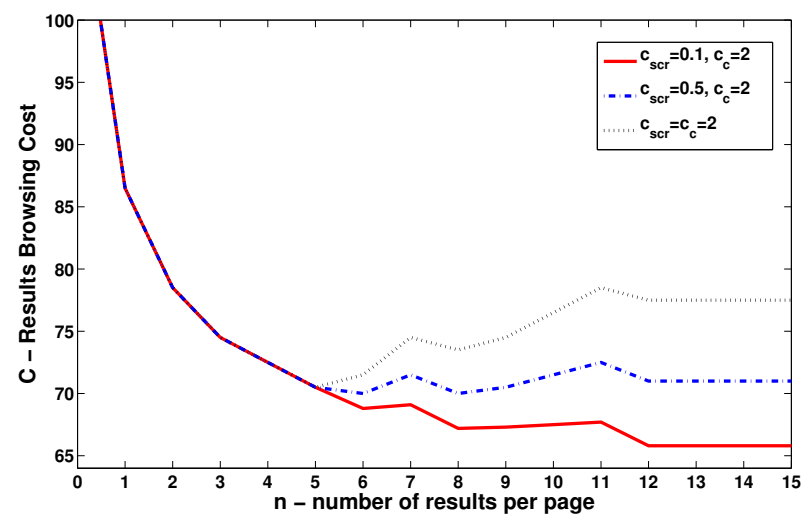

Fig. 3. An example of how the cost changes as the page size increases, when $m=25$ and $k \leq 6$ for the three different cases of $k . C_{s c r}$ versus $C_{c}$.

\section{Discussion \& Future Work}

We have derived a general cost model of result browsing, which provides an indication of how the total cost of interaction changes with SERP size (n), screen size $(\mathbf{k})$ and the associated interaction costs. In a desktop setting, as modeled above, where $\mathbf{k} \approx \mathbf{6}$ and the cost of click $\mathbf{C}_{\mathbf{c}}$ is generally larger than the scroll cost $\mathbf{C}_{\mathbf{s c r}}$, it is likely that the total cost is approximated by the blue dot dashed line and the red solid line shown in Figure 3. This is interesting because choosing a SERP size of $\mathbf{n}=\mathbf{1 0}$ (as done by most search engines when interacting from a PC), tends to be near or close to the minimum cost. While increasing the SERP 
size to beyond ten would lead to lower total costs, this is at a diminishing rate. In this model, we have assumed a fixed download cost, (within $\mathbf{C}_{\mathbf{c}}$ ). However, a more realistic estimate of this cost would be proportional to $\mathbf{n}$, such that $\mathbf{C}_{\mathbf{c}}(\mathbf{n})$, where a larger page takes longer to download. Another refinement of the model would be to condition scrolling on the number of results that need to be scrolled through; as users might find it increasingly difficult and cognitively taxing to scroll through long lists. Nonetheless, our model is still informative and a starting point for estimating the browsing costs. Future work, therefore, could: (i) extend the model to cater for these other costs in order to obtain a more accurate estimate of the overall cost, (ii) obtain empirical estimates for the different costs, on different devices (e.g., laptops, mobiles, desktops, tablets, etc.) as well as with different means of interaction (e.g., mouse with/without a scroll wheel, touchscreen, touchmouse, voice, etc.), and, (iii) incorporate such a browsing model into simulations, measures and analyses. A further extension would be to consider different types of layouts (e.g., grids, lists, columns, etc.) and different scenarios (e.g., finding an app on a tablet, mobile, etc.).

Acknowledgements: Thanks to all our tutorial participants who undertook this modelling exercise and helped us refine the model. Thanks to Diane Kelly \& Kathy Brennan for the numerous conversations about clicks and scrolls which led to this model \& title.

\section{References}

1. Azzopardi, L.: Modelling interaction with economic models of search. In: Proc. of the 37th ACM SIGIR conference. pp. 3-12 (2014)

2. Azzopardi, L., Kelly, D., Brennan, K.: How query cost affects search behavior. In: Proc. of the 36th ACM SIGIR conference. pp. 23-32 (2013)

3. Azzopardi, L., Zuccon, G.: An analysis of theories of search and search behavior. In: Proceedings of the 2015 International Conference on The Theory of Information Retrieval. pp. 81-90 (2015)

4. Baskaya, F., Keskustalo, H., Järvelin, K.: Time drives interaction: simulating sessions in diverse searching environments. In: Proc. of the 35th ACM SIGIR conference. pp. 105-114 (2012)

5. Baskaya, F., Keskustalo, H., Järvelin, K.: Modeling behavioral factors in interactive ir. In: Proc. of the 22nd ACM SIGIR conference. pp. 2297-2302 (2013)

6. Card, S.K., Moran, T.P., Newell, A.: The keystroke-level model for user performance time with interactive systems. Comm. of the ACM 23(7), 396-410 (1980)

7. Kashyap, A., Hristidis, V., Petropoulos, M.: Facetor: Cost-driven exploration of faceted query results. In: Proc. of the 19th ACM CIKM. pp. 719-728 (2010)

8. Kelly, D., Azzopardi, L.: How many results per page?: A study of serp size, search behavior and user experience. In: Proc. of the 38th ACM SIGIR Conference. pp. 183-192. SIGIR '15 (2015)

9. Pirolli, P., Card, S.: Information foraging. Psych. Review 106, 643-675 (1999)

10. Russell, D.M., Stefik, M.J., Pirolli, P., Card, S.K.: The cost structure of sensemaking. In: Proceedings of the INTERACT/SIGCHI. pp. 269-276 (1993)

11. Smucker, M.D., Clarke, C.L.: Time-based calibration of effectiveness measures. In: Proc. of the 35th ACM SIGIR conference. pp. 95-104 (2012)

12. Smucker, M.D., Jethani, C.P.: Human performance and retrieval precision revisited. In: Proc. of the 33rd ACM SIGIR conference. pp. 595-602 (2010) 